\title{
Erratum to: Convergence analysis of Legendre spectral projection methods for Hammerstein integral equations of mixed type
}

\author{
Payel Das' ${ }^{1}$ - Mitali Madhumita Sahani² . \\ Gnaneshwar Nelakanti ${ }^{1}$
}

Published online: 30 January 2016

(C) Korean Society for Computational and Applied Mathematics 2016

\section{Erratum to: J. Appl. Math. Comput. (2015) 49:529-555 DOI 10.1007/s12190-014-0852-8}

The authors regret the fact that throughout the paper, we assumed $\left\|\mathcal{P}_{n}\right\|_{L^{2}} \leq p$, where $p$ is a constant independent of $n$, which is not true. Although it is true that, $\left\|\mathcal{P}_{n} x\right\|_{L^{2}} \leq p_{1}\|x\|_{\infty}$, where $p_{1}$ is a constant independent of $n$. In Lemma 2.2 and 2.4, we quoted that $\left\{\mathcal{P}_{n}^{G}: n \in \mathbb{N}\right\}$ and $\left\{\mathcal{P}_{n}^{C}: n \in \mathbb{N}\right\}$ are uniformly bounded in $L^{2}$-norm. We now rewrite this statements as " $\left\|\mathcal{P}_{n}^{G} x\right\|_{L^{2}} \leq r_{1}\|x\|_{\infty}$ and $\left\|\mathcal{P}_{n}^{C} x\right\|_{L^{2}} \leq r_{2}\|x\|_{\infty}$ ", where $r_{1}$ and $r_{2}$ are constants independent of $n$. To prove the estimates (2.28), (2.29)and (2.33), we have used the estimate $\left\|\mathcal{P}_{n}\right\|_{L^{2}} \leq p$. Those estimates can be easily obtained by using the estimate $\left\|\mathcal{P}_{n} x\right\|_{L^{2}} \leq p_{1}\|x\|_{\infty}$ also.

The authors would like to apologize for any inconvenience caused.

The online version of the original article can be found under doi:10.1007/s12190-014-0852-8.

$\triangle$ Payel Das

dasPaye12@gmail.com

Mitali Madhumita Sahani

mitali.me@gmail.com

Gnaneshwar Nelakanti

gnanesh@maths.iitkgp.ernet.in

1 Department of Mathematics, Indian Institute of Technology, Kharagpur 721302, India

2 Department of Mathematics, School of Applied Sciences, KIIT University, Bhubaneswar 751024, India 